\title{
Simulation Method of SAR Raw Echo for Urban Scene
}

\author{
Yingxi Zheng, Bo Zhou, Zhulin Zhong, and Ming Lv \\ Research Institute of Electronic Science and Technology of \\ University of Electronic Science and Technology of China \\ Chengdu, Sichuan Province, China \\ zhengyingxi@uestc.edu.cn
}

\begin{abstract}
In this paper, a SAR raw data simulation method for urban scene is presented based on the hypothesis that urban area is a set of vertical buildings placed over a random rough dielectric terrain. Facet model and Kirchhoff approach appropriately including multiple-scattering effects are adopted here to compute scattering coefficients in the scattering model, which operates in twodimensional Fourier transformed domain. Methods of computing the scattering coefficients in different conditions are discussed in detail. Subsequently, the computational formulas and steps are also provided. It is known that the scattering model can effectively simulate the urban scene. The proposed simulation method of SAR raw echo turns out to be valid through simulation and analysis of the imaging to raw echo.
\end{abstract}

Keywords: SAR; facet; raw data simulation.

\section{Introduction}

SAR, one of the advanced techniques of radar imaging was developed in 1970s, which is applied widely in many areas such as military, ocean, agriculture and so on. Software simulation, which produces simulative echo and images, is a very important and economical method in research of SAR systems ${ }^{[1]}$.

Franceschetti ${ }^{[2]}$ shows a ground scene based digital elevation model (DEM) by establishing SARAS simulation system. A computing method of backscattered field of building scenes and some formulas are also provided in [3]

In this paper the methods of facet model and Kirchhoff approach are used to compute scattering coefficient based on the analysis to backscattering field and backscattering coefficient based upon [3].

\section{Scattering Model of Building}

Urban scene is supposed as a set of vertical buildings on a random rough dielectric terrain here. In case of just an isolated cube building for convenient analysis, whose geometrical figure is showed in fig1. Its vertical surface is parallel with axis $\mathrm{Z}$ and the bottom side is $\mathrm{Z}=0$. 


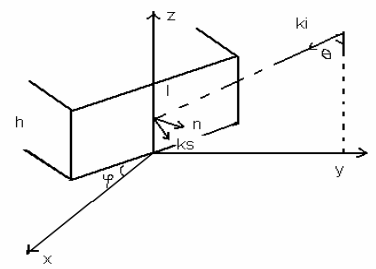

Fig. 1. Geometrical relation between building and ground

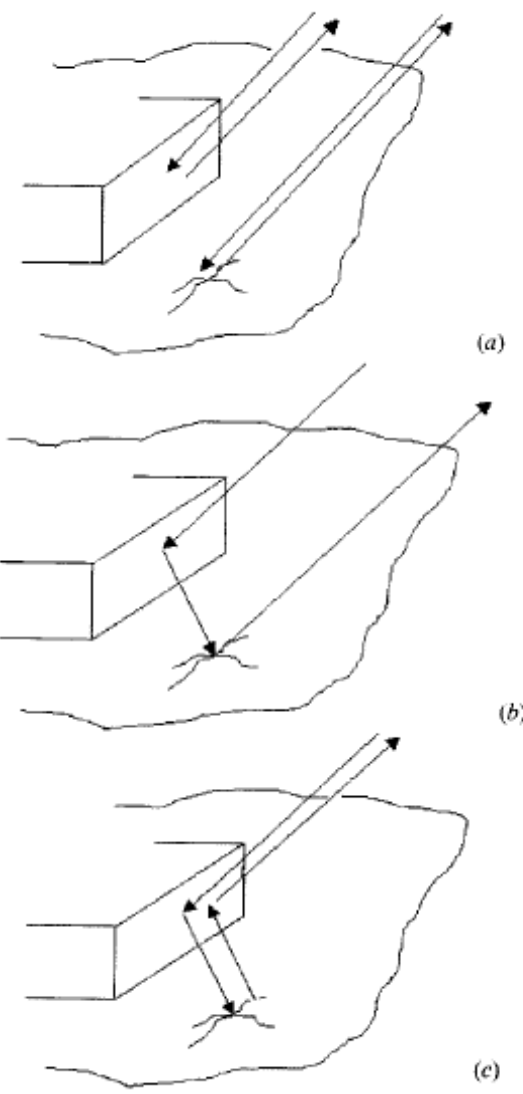

Fig. 2. (a) single, (b) double, and (c) triple scattering

In [4] the author analyzes the multiple scattering model and the conditions in point in detail, the relative conclusions of which are cited here directly. Superposition of first-, second-, and third-order contributions fully represent the scattered field; higher order mutual interactions do not give any contribution to the backscattered field to the radar antenna because the wall surface is supposed flat. In order to account for multiple scattering between buildings and terrain, we use Geometric optics $(\mathrm{GO})$ to evaluate the field 
reflected by the smooth wall toward the ground (first bounce) or the sensor (second or third bounce), and PO (Physical optics) or GO (depending on ground surface roughness) to evaluate the field scattered by the ground toward the wall (first or second bounce) or the sensor (second bounce).

In the prevenient literature there are many researches about the computing method of backscattering field which has only single scattering. The method in [2] is employed in the paper. The statistic characteristic of backscattering coefficient of single scattering and double scattering has been given in [3] (Table III, Table IV). Below the method of computing scattering coefficient in double and triple scattering based upon single facet will be deduced. The single-[fig.2 (a)], double-[fig.2 (b)], triple[fig.2(c)] scattering are illuminated as follows.

\section{Facet Model}

Facet Model is physical model of simulating ground scattering characteristics. The natural ground scene can be described by amounts of small facets which are tangent to the surface of scenes. The electromagnetic field scattering characteristics of the ground scene result from coherent superposition of backscattering field of facet ${ }^{[5]}$. The electromagnetic field scattering characteristics of facet is decided according to the roughness degree ${ }^{[6]}$ of the facet surface and medium material ${ }^{[7]}$ The dimension of facet is bigger than the signal wavelength and less than resolution cell of radar so far ${ }^{[3]}$.

\section{The Computation Method of Scattering Coefficient}

\subsection{Scattering Coefficient $S_{w g}$ from Walls to the Ground}

Because the relative is not backscattering between the direction of incident wave and scattering wave, namely $\vec{k}_{s} \neq-\vec{k}_{i}$, and the facet scattering coefficient computation formula can be not used directly here in literature [3] and need to be deduced again.

According to Kirchhoff approach theory scattering field physical optics of each facet can be deem approximately:

$$
\vec{E}_{s}(\vec{r})=\frac{j k e^{j k r}}{4 \pi r} E_{0}\left(I-\vec{k}_{s} \vec{k}_{s}\right) \cdot \vec{F}\left(\vec{k}_{i}, \vec{e}_{i}, \vec{n}\right) \cdot I_{s}
$$

Wherein

$$
\begin{aligned}
& \vec{F}\left(\vec{k}_{i}, \vec{e}_{i}, \vec{n}\right)=-\left(\vec{e}_{i} \cdot \vec{q}_{i}\right)\left(\vec{n} \cdot \vec{k}_{i}\right) \vec{q}_{i}\left(1-R_{p}\right)+\left(\vec{e}_{i} \cdot \vec{p}_{i}\right)\left(\vec{n} \times \vec{q}_{i}\right)\left(1+R_{q}\right) \\
& +\left(\vec{e}_{i} \cdot \vec{q}_{i}\right)\left(\vec{k}_{s} \times\left(\vec{n} \times \vec{q}_{i}\right)\right)\left(1+R_{p}\right)+\left(\vec{e}_{i} \cdot \vec{p}_{i}\right)\left(\vec{n} \cdot \vec{k}_{i}\right)\left(\vec{k}_{s} \times \vec{q}_{i}\right)\left(1-R_{q}\right)
\end{aligned}
$$

where $\vec{r}$ is the vector from antenna to facet, $k$ is wavenumber, $I$ is unit matrix, $\vec{E}_{s}(\vec{r})$ is scattering field strength of facet along the direction of $\vec{k}_{s}, \vec{q}_{i}=\frac{\vec{k}_{i} \times \vec{n}}{\left|\vec{k}_{i} \times \vec{n}\right|}$, 
$\vec{p}_{i}=\vec{q}_{i} \times \vec{k}_{i}, R_{p}$ and $R_{q}$ are respectively polarized Fresnel reflection coefficients along the direction of $\vec{p}_{i}$ and $\vec{q}_{i}$. $\vec{F}$ reflects the electromagnetism characteristics of polarization and facet surface and $I_{s}$ reflects the shape of facet ${ }^{[2]}$.

Suppose horizontal polarization direction unit vectors of incident field and scattering field are $\vec{e}_{i h}=\frac{\vec{z} \times \vec{k}_{i}}{\left|\vec{z} \times \vec{k}_{i}\right|}$ and $\vec{e}_{s h}=\frac{\vec{z} \times \vec{k}_{s}}{\left|\vec{z} \times \vec{k}_{s}\right|}$ respectively, and the vertical polarization direction unit vectors are $\vec{e}_{i v}=\vec{e}_{i h} \times \vec{k}_{i}$ and $\vec{e}_{s v}=\vec{e}_{s h} \times \vec{k}_{s}$.

According to the geometrical relative in fig.1,

$$
\vec{k}_{i}=-\sin \theta \vec{y}-\cos \theta, \vec{n}=\sin \varphi \vec{x}+\cos \varphi \vec{y}
$$

Meantime, the scattering field strength along the lens reflection direction of incident wave is biggest because the surface of walls is flat. Here $\vec{k}_{s}$ is reflection direction and

$$
\vec{k}_{s}=\sin \theta \sin 2 \varphi \vec{x}+\sin \theta \cos 2 \varphi \vec{y}-\cos \theta \vec{z}
$$

The elements of scattering matrix

$$
S_{w g}=\left(\begin{array}{ll}
S_{h h} & S_{v h} \\
S_{h v} & S_{v v}
\end{array}\right)
$$

can be given,

$$
\begin{gathered}
S_{h h}=\frac{2 \sin \theta \cos \varphi\left(\cos ^{2} \theta \cos ^{2} \varphi R_{p}-\sin ^{2} \varphi R_{q}\right)}{\cos ^{2} \theta \cos ^{2} \varphi+\sin ^{2} \varphi} \\
S_{h v}=\frac{2 \sin \theta \cos \theta \sin \varphi \cos ^{2} \varphi\left(R_{p}+R_{q}\right)}{\cos ^{2} \theta \cos ^{2} \varphi+\sin ^{2} \varphi} \\
S_{v h}=\frac{-2 \sin \theta \cos \theta \sin \varphi \cos ^{2} \varphi\left(R_{p}+R_{q}\right)}{\cos ^{2} \theta \cos ^{2} \varphi+\sin ^{2} \varphi} \\
S_{v v}=\frac{2 \sin \theta \cos \varphi\left(-\sin ^{2} \varphi R_{p}+\cos ^{2} \theta \cos ^{2} \varphi R_{q}\right)}{\cos ^{2} \theta \cos ^{2} \varphi+\sin ^{2} \varphi}
\end{gathered}
$$

As $I_{s}=\int_{s} \exp \left[j\left(\vec{k}_{i}-\vec{k}_{s}\right) \cdot \vec{r}^{\prime}\right] d \vec{r}^{\prime}$ and $\vec{k}_{i}-\vec{k}_{s}$ are vertical to facet, $I_{s}=A$ and $A$ is the area of facet. 


\subsection{Scattering Coefficient in Other Conditions}

\subsubsection{Scattering Coefficient $S_{w r}$ from Walls to Radar}

It is similar to the condition from walls to the ground. Now let $-\vec{k}_{i}$ instead of $\vec{k}_{i},-\vec{k}_{i}$ instead of $\vec{k}_{s}, \vec{e}_{s h}$ instead of $\vec{e}_{i h}, \vec{e}_{i h}$ instead of $\vec{e}_{i v}$ and bring them into formula (2). The scattering matrix can be computed. Meantime, $I_{s}=A$ and $A$ is the area of facet.

\subsubsection{Scattering Coefficient $S_{g w}$ from the Ground to Walls}

There are two cases:

One: The scattering is backscattering when it is the second bound in triple scattering.

Two: When it is the first bound in double scattering, the normal vector of facet

\subsubsection{Scattering Coefficient $S_{g r}$ from the Ground to the Sensor}

There are three cases:

One: When it is the second bound in double scattering, it can be computed by equation (2) directly.

Two: When it is the second bound in triple scattering, it belongs backscattering.

Three: When it is the first bound, it belongs backscattering.

\subsection{Computation of Multiple-Scattering Coefficients}

In conclusion from that, the computation of double scattering and triple scattering matrixes are as follows,

$$
\begin{gathered}
S=S_{g w r}+S_{w g r}=S_{w r} S_{g w}+S_{g r} S_{w g} \\
S=S_{w g w r}=S_{w r} S_{g w} S_{w g}
\end{gathered}
$$

\section{Echo Generation Model}

We consider that echo is get by targets' backscattering coefficient through a system function $h(x, r)$, namely $s(x, r)=\sigma(x, r) \otimes h(x, r)$, and $h(x, r)$ is expressed ${ }^{[8]}$.

$$
h(x, r)=w_{a}(x) w_{r}(r) \operatorname{rect}\left\{\frac{2[r-R(x)]}{C}\right\} e^{j \frac{4 \pi[r-R(x)]}{\lambda}} e^{j \frac{4 \pi K[r-R(x)]^{2}}{C^{2}}}
$$

Where $\sigma(x, r)$ is backscattering coefficient, $w_{a}(\cdot)$ is azimuth antenna pattern, $w_{r}(\cdot)$ is distance antenna pattern, $\operatorname{rect}(\cdot)$ is rectangular window function, $R(x)$ is the distance 
between targets and radar, $\mathrm{C}$ is light velocity, $\lambda$ is wavelength and $K$ is FM-ratio of line frequency modulation signal (LFM).

The flow of echo simulation is as follows.

One: Generate building scene DEM data under background of Gauss random distribution.

Two: Compute the first scattering coefficient and the mean of scattering coefficient of the same distance cells to the ground facet.

Three: Compute the second scattering coefficient and the mean of scattering coefficient of the same distance cells to the ground facet and building facet.

Four: Compute the third scattering coefficient and the mean of scattering coefficient of the same distance cells to the ground facet and building facet.

Five: Perform multiplication between 2-D FFT of scattering coefficient $\sigma(x, r)$ and 2-D FFT of system function $h(x, r)$, which the result is $S(k, f)$.

Six: The raw echo $s(x, r)$ is provided by operating 2-D FFT to $S(k, f)$

\section{Simulation Results}

Table 1. The system parameters of airborne SAR simulation

\begin{tabular}{|l|l|}
\hline Parameter & Value \\
\hline Height & $20000 \mathrm{~m}$ \\
\hline Wavelength & $0.03 \mathrm{~m}$ \\
\hline Velocity of airplane & $250 \mathrm{~m} / \mathrm{s}$ \\
\hline Horizontal wave width & $4.3^{\circ}$ \\
\hline Depression angle & $25^{\circ}$ \\
\hline Vertical wave width & $7.3^{\circ}$ \\
\hline Bandwidth & $40 \mathrm{MHz}$ \\
\hline Sampling rate & $50 \mathrm{MHz}$ \\
\hline Resolution & $5 \mathrm{~m} \times 5 \mathrm{~m}$ \\
\hline
\end{tabular}

Table 2. The parameters of ground scene

\begin{tabular}{|l|l|l|}
\hline Parameter & \multicolumn{2}{|l|}{ Value } \\
\hline Building height (h) & $25 \mathrm{~m}$ & $40 \mathrm{~m}$ \\
\hline Building width (b) & $25 \mathrm{~m}$ & $40 \mathrm{~m}$ \\
\hline Building length (l) & $25 \mathrm{~m}$ & $40 \mathrm{~m}$ \\
\hline Angle between wall and flight direction $(\boldsymbol{\varphi})$ & $0^{\circ}$ & $45^{\circ}$ \\
\hline building and ground dielectric constant $\mathcal{E}_{r}$ & 4 & \\
\hline $\begin{array}{l}\text { ground height obeys gauss random distribution } \\
\text { and coarseness degree: } k \sigma\end{array}$ & 6.28 \\
\hline polarization mode & VV \\
\hline
\end{tabular}

The scattering figures are fig.3, fig.4, fig.5, fig.6 and image of raw signal is fig.7. 


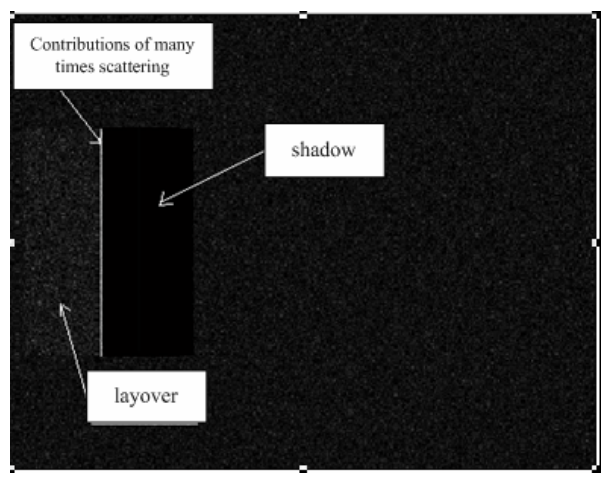

Fig. 3. Scattering figure when $h=40 \mathrm{~m}, \mathrm{~b}=40 \mathrm{~m}, 1=25 \mathrm{~m}, \varphi=0^{\circ}$

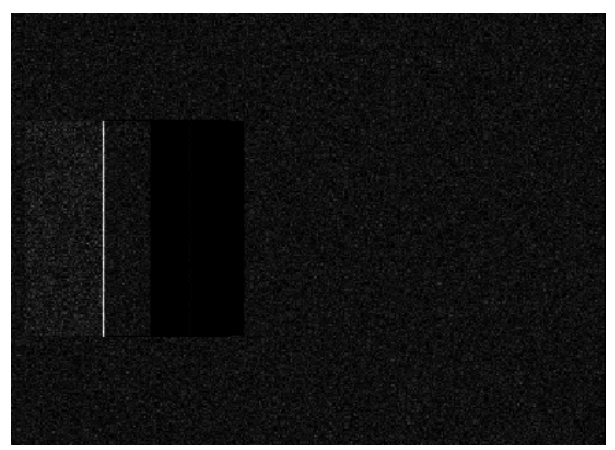

Fig. 4. Scattering figure when $\mathrm{h}=40 \mathrm{~m}, \mathrm{~b}=40 \mathrm{~m}, 1=40 \mathrm{~m}, \varphi=0^{\circ}$

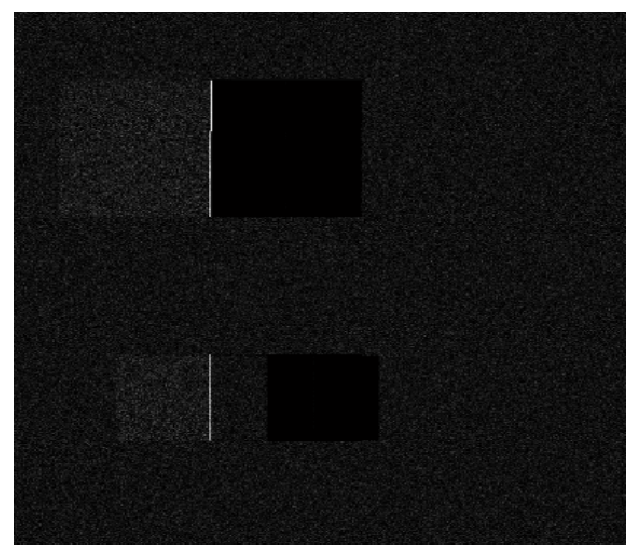

Fig. 5. Building scattering figure when $h=40 \mathrm{~m}, \mathrm{~b}=40 \mathrm{~m}, \mathrm{l}=25 \mathrm{~m}, \varphi=0^{\circ}$ and $\mathrm{h}=25 \mathrm{~m}, \mathrm{~b}=25 \mathrm{~m}, \mathrm{l}=25 \mathrm{~m}$, $\varphi=0^{\circ}$ 


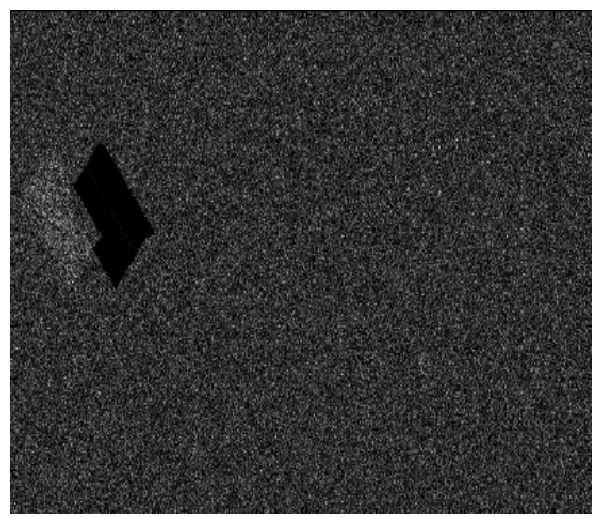

Fig. 6. Scattering figure when $\mathrm{h}=40 \mathrm{~m}, \mathrm{~b}=40 \mathrm{~m}, \mathrm{l}=40 \mathrm{~m}, \varphi=45^{\circ}$

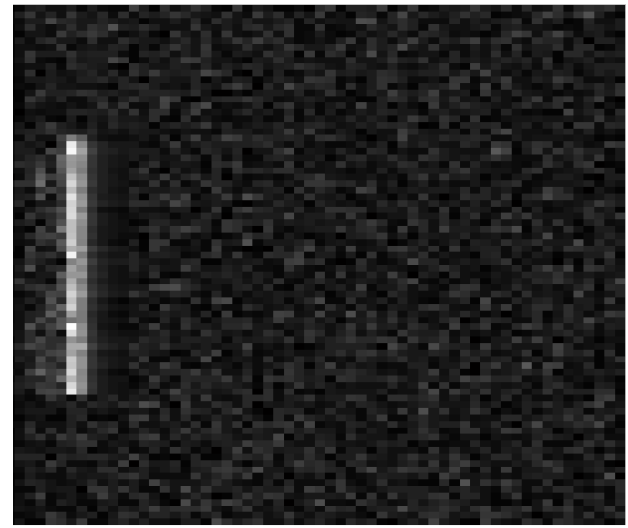

Fig. 7. Image when $h=40 \mathrm{~m}, \mathrm{~b}=40 \mathrm{~m}, \mathrm{l}=25 \mathrm{~m}, \varphi=0^{\circ}$

From fig.2 and fig.3, we can obviously see layover from left to right, subsequently light scattering lines resulting from multiple scatterings of walls and shadow and special phenomenon of SAR image. Meanwhile, the locations of layover and shadow are relative to the length of the building. We can see from fig. 7 that image is approach to corresponding scattering coefficient in fig. 3.

\section{Conclusion}

The paper gives the formulas of computing scattering coefficient from wall to ground in double and triple scattering based on facet building according to Kirchhoff approach and facet model. At the same time, the scattering coefficients and echoes of building scenes with different parameters are also simulated. The result of test shows hat the simulative method can response the special characteristics of SAR image, such 
as layover, shadow, azimuth base point and so on. Moreover, the method can simulate raw signals adapted various SARs to different roughness degrees, different dielectrics, different building shapes, different polarizations and so on.

Acknowledgments. The authors thank for Li Yangyuan' helpful advices.

\section{References}

1. Jinrao, S., Hong, S.: Simulation for natural scene SAR image. Rada science and technique 1(4), 200-214 (2003)

2. Franceschetti, G., Migliaccio, M., Riccio, D.: SARAS: a synthetic aperture radar (SAR) raw signal simulator. IEEE Trans, Geoscience and Remote Sensing 30(1), 110-123 (1992)

3. Franceschetti, G., Iodice, A., Riccio, D.: A canonical problem in electromagnetic backscattering from buildings. IEEE Trans, Geoscience and Remote Sensing 40(8), 1787-1801 (2002)

4. Franceschetti, G., Iodice, A., Riccio, D., et al.: SAR raw signal simulation for urban structures. IEEE Trans, Geoscience and Remote Sensing 41(9), 1986-1995 (1986)

5. Feng, W.: Raw Data Simulation for DistributionTargets of Space-borne SAR, mater degree paper (2003)

6. Jianchao, F., et al.: Microwave Remote Sensing-Radar Remote Sensing and Scattering and Radiation Theory of Face Targets. Science Press, BJ (1987)

7. Jie, C., Yinqing, Z., Chunsheng, L.: Research of simulation method for natural ground scene of space-borne SAR. Electronic transaction 29(9), 1202-1205 (2001)

8. Yongtan, L., et al.: Radar Imging Technique, Ha Er Bin Industry Universtity Press, 10 (1990) 